\title{
A SIMPLE METHOD OF DEW MEASUREMENT
}

\section{By A. Potvin}

\section{Dominion Forest Service, Ottawa, Ontario}

As a meteorological phenomenon, dew has not been given the same attention as other weather factors. While this form of water vapour condensation does not appear to affect plant life to the same extent as rain, temperature or wind, it nevertheless influences vegetation to a certain degree. Agriculture and forestry are consequently the two sciences that should benefit most from studies of dew formation.

While foresters on this continent recognize the value of dew as a contributing factor in forest fire control, this factor has not yet been incorporated in any forest fire-danger rating system. This omission can be attributed in part to inadequate methods of measurement, as well as to insufficient knowledge of the effect of dew on forest fuels.

Until now, two main methods have been used to estimate the amount of dew formed during the night; the weighing method and the optical method. ${ }^{1}$ This latter system is of quite recent development. Both these methods have drawbacks which limit their usefulness for accurate dew measurement, although the optical method appears to be a definite improvement over the weighing method. In the summer of 1948 a simple means of dew measurement was devised by the author, and developed on the Forest. Fire Hazard Research Survey headed by J. C. Macleod, in the Whiteshell Forest Reserve, Manitoba. It might be called the direct measurement method.

The weighing method consists in weighing some absorbent material, such as a Leick plate, that has been exposed outdoors overnight and deducting from this weight the weight before exposure. The unreliability of this method arises primarily from the fact that uniformity of the absorbent material is practically impossible of achievement, owing to the infinite variations in the pores of the substance used. Other disadvantages are the time required to dry out the material, the changes that take place in its cell structure, the tendency of the pores to clog after long periods of exposure, and the possibility of errors due to poorly adjusted balances or inaccurate readings of the scale. Furthermore, since evaporation starts soon after sun-up, the weighings must be taken at this early hour; this inconvenience alone would make it very unpopular.

To estimate by the visual method the amount of dew formed overnight, wooden blocks, coated with a special paint are used. The patterns formed by water vapour condensation on these blocks vary with the amount deposited; thus, by comparing the appearance of these blocks with a set of 10 photographs illustrating the progressive stages of surface transformation brought about by varying amounts of dew, a numerical rating can be given.

1. Duvdevani, S. "An Optical Method of Dew Estimation". Quarterly Journal of the Royal Meteorological Society, Vol. 73, 1947. 
The principal drawback to this method is the fact that it depends to such a great extent on the personal equation. The difference between a given pattern and those adjoining it in the series is such that different observers may easily differ in their judgment as to which pattern applies. While discrepancies arising from this source may, in the case of a number of observers, balance out over a prolonged period, where one observer is concerned the error is rather liable to be cumulative. Moreover, the blocks are difficult to prepare uniformly, are affected by exposure, and are subject to fungus attack.

The direct measurement method developed by the Dominion Forest Service involves the use of a sheet of glass on which the dew collects and from which it drips into graduated gauge. Glass was used in preference to other materials because of its high rate of infra-red radiation which allows it to cool more rapidly down to the dew point. As the amount collected is read directly from the graduate, no conversion scale or table is needed.

From the initial tests carried out this year, it was found that readings were scarcely, if at all, affected by the thickness of the glass used, but that the angle at which the glass plate was placed was of considerable importance. Thus, a sheet of glass suspended vertically yielded, on the average, only 60 per cent of the total recorded when the instrument was set at a $45^{\circ}$ angle in relation to the ground. This last setting was adopted as standard after it proved to yield the greatest amount of dew while still allowing for good drainage. Double diamond glass plates, 1 foot square, were chosen to ensure easy duplication.

During the three months that the trials were run, the maximum amount of dew collected in one night from this exposed surface was $26 \mathrm{ccs}$; the average for all the nights on which dew formation took place was 10.5 ccs.

This method of dew measurement has no complex features, requiring only a sheet of glass, a support, and a glass gauge graduated in cubic centimeters. The total cost of the instrument is about $\$ 1.50$.

A further analysis will be undertaken to study the effect of forest canopy on dew, the effect of dew on different fuels and its value as a retardant in forest fire control. 